Research Paper

\title{
Wnt5a Suppresses $\beta$-catenin Signaling during Hair Follicle Regeneration
}

\author{
Yizhan Xing, Xiaogen Ma, Haiying Guo, Fang Deng, Jin Yang ${ }^{\bowtie}$, Yuhong Li $^{\bowtie}$ \\ Department of Cell Biology, College of Basic Medical Sciences, Third Military Medical University, Chongqing, China. \\ $\triangle$ Corresponding authors: Yuhong Li: Tel: +86 23 68752253, E-mail: liyuhongtmmu@hotmail.com Or Jin Yang: Tel: +86 23 68752251, E-mail: \\ zhiwen@hotmail.com. Department of Cell Biology, College of Basic Medical Sciences, Third Military Medical University, Gaotanyan Street No. 30, Shapingba \\ District, Chongqing, China, 400038.
}

(C) Ivyspring International Publisher. Reproduction is permitted for personal, noncommercial use, provided that the article is in whole, unmodified, and properly cited. See http://ivyspring.com/terms for terms and conditions.

Received: 2016.03.18; Accepted: 2016.07.08; Published: 2016.07.18

\begin{abstract}
Hair follicles display periodic growth. Wnt signaling is a critical regulator for hair follicle regeneration. Previously, we reported that $\mathrm{Wnt} 5 \mathrm{a}$ inhibits the telogen-to-anagen transition of hair follicles, but the mechanism by which this process occurs has not yet been reported. Here, we determined the expression patterns of Wnt signaling pathway molecules by quantitative reverse transcription polymerase chain reaction, western blot, and immunohistochemistry and found that $\beta$-catenin signaling was suppressed by $\mathrm{Wnt} 5 \mathrm{a}$. We then compared the phenotypes and expression patterns following $\beta$-catenin knockdown and $W n t 5 a$ overexpression during hair follicle regeneration induced by hair depilation and observed similar patterns. In addition, we performed a rescue experiment in the JB6 cell line and found that the inhibitory effect of Wnt5a on cell proliferation could be rescued by the addition of Wnt3a. Our data reveal that Wnt5a suppresses the activation of $\beta$-catenin signaling during hair follicle regeneration.
\end{abstract}

Key words: Wnt signaling pathway, $\beta$-catenin, Wnt5a, hair follicle, regeneration.

\section{Introduction}

Hair follicles are one of the accessory mini-organs of the skin. Hair follicles begin to form during embryonic development and finish morphogenesis postnatally. Over the lifetime of an individual, hair follicles undergo periodic growth [1]. The hair cycle consists of anagen, catagen and telogen phases. Dysfunction of the hair cycle results in hair follicle-associated diseases, including hair loss and hirsutism [2]. Many signaling pathways are reported to play roles in the regulation of the hair cycle, including the fibroblast growth factor [3], bone morphogenetic proteins [4], and Wnt (wingless-type mouse mammary tumor virus integration site) pathways [5].

Wnt proteins are a family of secreted proteins. Humans and mice possess 19 types of Wnt proteins. Wnt proteins mediate physiological effects via the canonical Wnt signaling pathway or through non-canonical Wnt signaling pathways. The canonical Wnt signaling pathway is also termed the
Wnt/ $\beta$-catenin signaling pathway because its activation is usually accompanied by the translocation of $\beta$-catenin from the plasma to the nucleus. Non-canonical Wnt signaling pathways are grouped into several categories for clarity and simplicity and include the Wnt/planar cell polarity (PCP) signaling pathway, the Wnt-cGMP/Ca ${ }^{2+}$ signaling pathway, and the Wnt-receptor tyrosine kinase-like orphan receptor 2 signaling pathway [6]. Non-canonical Wnt signaling pathways are diverse and, in many cases, less well-characterized. The relationship between the canonical Wnt signaling pathway and non-canonical Wnt signaling pathways is not clearly defined.

Wnt signaling pathways play critical roles in embryonic development, tumorigenesis, and stem cell differentiation [7]. Hair follicle morphogenesis and regeneration would be impacted severely if any of the molecules in the canonical Wnt signaling chain were blocked [8]. Some Wnt ligands have been reported to regulate the hair cycle, including Wnt3a, Wnt7a, and 
Wnt7b $[9,10]$. Previously, we reported that Wnt10b promotes hair follicle growth via the canonical Wnt signaling pathway [11] and that the overexpression of Wnt10b induces hair follicle regeneration in vivo [12]. We also found that the overexpression of Wnt5a suppresses the transition of hair follicles from telogen to anagen [13]. However, the signaling pathway through which Wnt5a signals in hair follicle regeneration remains to be clarified. Here, we found that overexpressed Wnt5a suppressed the expression and translocation of $\beta$-catenin during hair follicle regeneration and that the phenotype and expression patterns were similar to those observed upon knockdown of $\beta$-catenin. Our data prove that Wnt5a suppresses hair follicle regeneration by suppressing the activation of the canonical Wnt signaling pathway.

\section{Materials and Methods}

\section{Animals}

C57BL/6 mice were housed in the Laboratory Animal Center of Third Military Medical University, Chongqing, China. All of the experimental protocols were approved by the Research Committee of Third Military Medical University. Female mice aged 7-8 weeks were used for experiments. Mice were kept in a 12-hour light/dark cycle and fed a normal diet.

\section{Intradermal administration of adenoviruses}

Adenoviruses, including AdWnt5a (mouse Wnt5a cDNA encoded in adenovirus vector), AdSimBC (siRNA targeting $\beta$-catenin encoded in adenovirus vector) and AdGFP (green fluorescence protein encoded in adenovirus vector, used as control), were gifts from Dr. Tong-Chuan $\mathrm{He}$, University of Chicago, USA. The adenoviruses were propagated in HEK293 cells as previously described [14]. The dorsal skin of mice was depilated, and $50 \mu \mathrm{l}$ of adenovirus vector (AdWnt5a or AdGFP) was injected intradermally along the median dorsal line of the skin. Dorsal skins were harvested for histological analysis and immunostaining at the designated time points after administration.

\section{Immunohistochemical analysis}

Immunohistochemical analysis was performed on paraffin-embedded sections from dorsal skin. Sections were incubated with primary antibodies: rabbit anti-Wnt5a (1:100, Santa Cruz, USA), mouse anti- $\beta$-catenin (1:100, Santa Cruz, USA), rabbit anti-Dsh (1:100; Santa Cruz, USA), goat anti-Ki67 (1:100, Abcam, UK), goat anti-lef1 (1:100, Santa Cruz, USA), mouse anti-AE15 (1:4, provided by TT Sun, New York University, USA). HRP-labeled secondary antibodies (Zhongshan Goldenbridge, China) were used. A DAB kit (Zhongshan Goldenbridge, China) was used to detect the expression of antigens according to the manufacturer's protocol.

\section{Quantitative real-time reverse transcription polymerase chain reaction (qRT-PCR)}

Skin tissue samples were collected from C57BL/ 6 mice at 8 weeks (telogen), 7 days after hair depilation at 8 weeks (middle anagen), or 7 days after hair depilation and intradermal injection of AdWnt5a at 8 weeks (prolonged telogen). Total RNA was extracted, the cDNA was synthesized with ReverTra Ace-a- kit (Toyobo, Japan), and qRT-PCR amplification was performed using a SuperGreen PCR kit II (CapitalBio, China) according to the manufacturer's protocol. Differences between measurements were evaluated using Student's t-test. For all statistical tests, a P-value of $<0.05$ was defined to be statistically significant.

\section{Western blot analysis}

Denatured protein extracted from skin tissue samples was loaded on a 10\% SDS-PAGE gel, electrophoresed, and then transferred onto a PVDF membrane. PVDF membranes were blocked with 5\% fat-free milk, and the membranes were then probed with rabbit anti-Wnt5a (1:1,000; Abcam, USA), goat anti-Frizzled (1:1,000, Santa Cruz, USA), rabbit anti-Dvl3 (1:1,000, Santa Cruz, USA), mouse anti- $\beta$-catenin (1:1,000; Santa Cruz, USA), goat anti-Lef1 (1:1,000, Santa Cruz, USA), and mouse anti-GAPDH antibody (1:500, Zhongshan Goldenbridge, China) at $4^{\circ} \mathrm{C}$ overnight. Blots were then incubated with secondary antibody. Peroxidase activity on the membrane was visualized on X-ray film using the ECL western blotting detection system.

\section{Cell culture and cell cycle analysis}

JB6 cells were cultured in DMEM (Hyclone, USA) containing 10\% FBS (Gibco, USA). For cell cycle analysis, JB6 cells were plated onto 6-well plate at a confluence of $70 \%$ to $80 \%$. After 6 hours, Wnt5a protein $(4 \mu \mathrm{g}$, R\&D, USA), Wnt3a protein ( $1 \mu \mathrm{g}$, R\&D, USA) or control dissolvent was added to the culture medium. Cells were harvested after 24 hours, and cell cycles were analyzed under a fluorescence-activated cell sorter according to standard protocols.

\section{Methyl thiazolyl tetrazolium (MTT) assay}

JB6 cells were plated onto 96-well plates. Six hours later, Wnt5a protein $(0.2 \mu \mathrm{g}$, R\&D, USA), Wnt3a protein $(0.1 \mu \mathrm{g}, \mathrm{R} \& \mathrm{D}, \mathrm{USA})$ or control dissolvent were added to the culture medium. Twenty-four hours later, $20 \mu \mathrm{l}$ of $5 \mathrm{mg} / \mathrm{ml}$ MTT was added to each well. Four hours later, the culture medium was aspirated out, $150 \mu$ l DMSO (Invitrogen, USA) was added, and 
the plates were shaken for 10 minutes. The absorbance value was determined by an enzyme-linked immunoassay at $490 \mathrm{~nm}$. Significant differences were evaluated using a t-test, and $\mathrm{p}<0.05$ was considered statistically significant.

\section{Results}

\section{Wnt5a suppresses the expression of $\beta$-catenin}

Three days after injection of adenovirus, Wnt5a was expressed in both hair follicle cells and interfollicular cells of AdWnt5a-injected skin (Fig. 1b), whereas no Wnt5a expression was observed in AdGFP-injected skin (Fig. 1a). In addition, AdWnt5a was used to treat JB6 cells in vitro. Thirty-six hours after treatment, Wnt5a was expressed in the skin samples of the AdWnt5a-treated group, whereas Wnt5a was not expressed in the samples from the AdGFP-treated group (Fig. 1c).

To determine the mechanism by which Wnt5a suppresses hair follicle regeneration, the expression of a number of key molecules in the Wnt signaling pathway was analyzed by qRT-PCR and western blot. Compared with middle anagen skin, the gene expression levels of Rhoa, Ror2, and Frizzled2 in prolonged telogen skin were lower. However, compared with normal telogen skin, the gene expressions of Rho2 and Frizzled2 in prolonged telogen skin were higher (Fig. 2a-2c). Compared with middle anagen skin, the protein expression of Wnt5a was increased, whereas the protein expression of $\beta$-catenin and Lef1 was decreased (Fig. 2d).

Next, the expression patterns of $\beta$-catenin, Frizzled and Ki67 were determined by
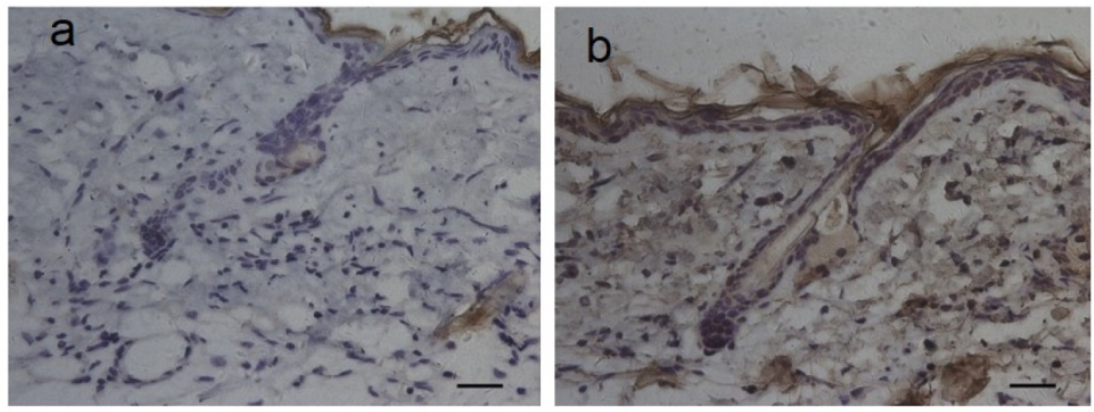

immunohistochemistry in hair-depilated dorsal skin of C57 mice. Seven days after AdWnt5a injected into the skin, the expression of $\beta$-catenin in hair follicles was not detectable, whereas the expression of $\beta$-catenin was observed in the control group (Fig. 3a, 3d). In hair follicles, the expression of Frizzled, a Wnt receptor, was observed in both the AdWnt5a and AdGFP groups (Fig. 3b, 3e). The expression of the proliferation marker Ki67 was also observed in both the AdWnt5a and AdGFP groups (Fig. 3c, 3f). The number of Ki67 positive cells in the AdWnt5a group was significantly lower than in the AdGFP group. These data indicate that Wnt5a may suppress the activation of the $\mathrm{Wnt} / \beta$-catenin pathway.

\section{Knockdown of $\beta$-catenin produces a similar phenotype and gene expression pattern as overexpression of Wnt5a}

When AdSimBC was injected intradermally into the hair-depilation induced skin, hair follicle regeneration was prolonged by approximately 40 days (Fig. 4a). At 7 days after AdSimBC injection, HE staining demonstrated that the entire injected area remained in telogen, whereas the adjacent area had already entered anagen (Fig. 4b). At 50 days after AdSimBC injection, the hair follicles in the injected area were normal in morphology (Fig. 4a), and all four hair types were found among the regenerated hairs (Fig. 4c-4e). These data demonstrate that the effect durations of AdSimBC can last for about 40 days and confirm that canonical Wnt signaling is an activator of hair follicle regeneration.

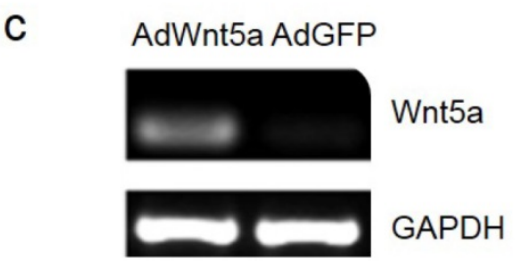

Figure 1. Expression of Wnt5a after injection of AdWnt5a. (a-b) The dorsal skin of C57 mice at postnatal day 56 was depilated, and AdGFP or AdWnt5a were injected into the depilated skin. Expression of Wnt5a was determined by immunohistochemistry 3 days after injection. (a) AdGFP-treated group. (b) AdWnt5a-treated group. (c) Cultured JB6 cells were treated with AdWnt5a or AdGFP. The expression of Wnt5a was determined by RT-PCR 36 hours after treatment. Scale bar $=25 \mu \mathrm{m}$. 

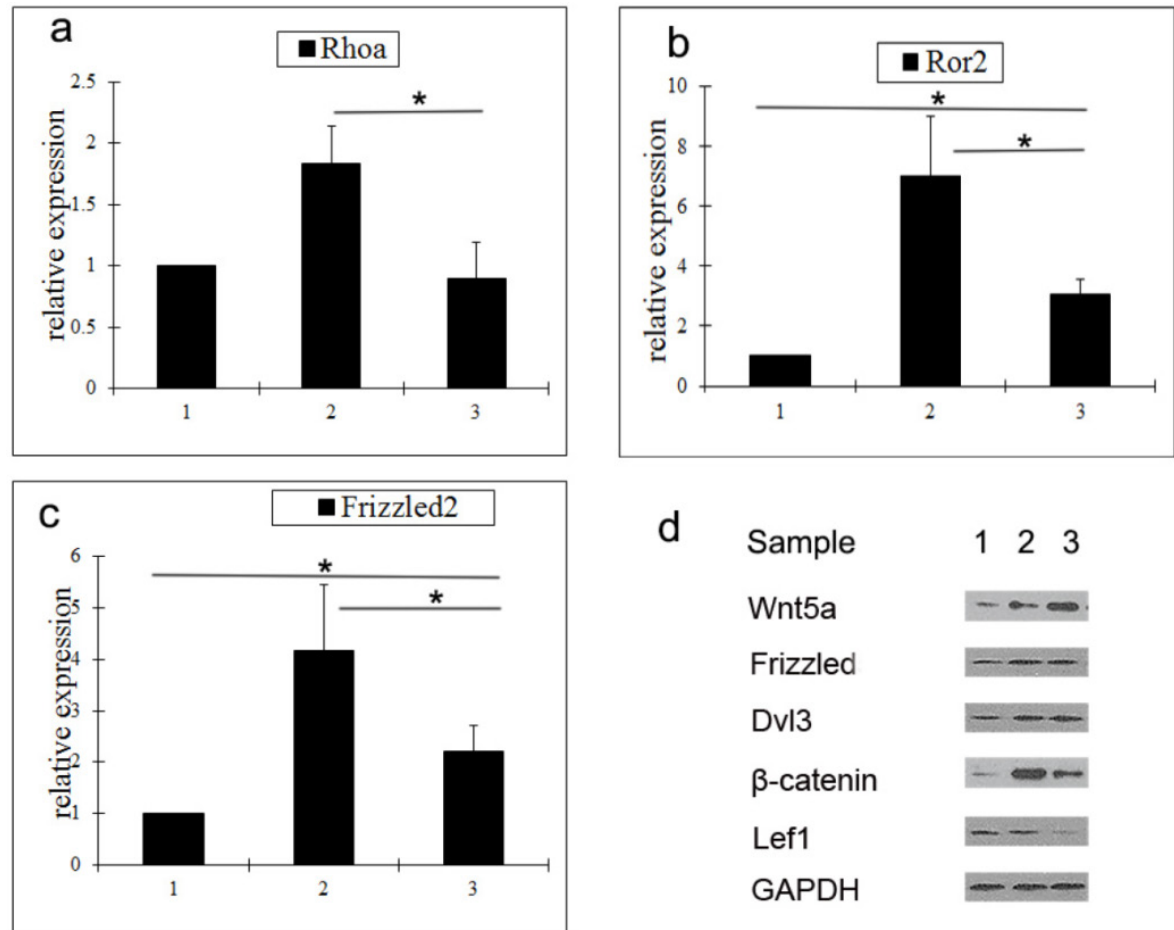

Figure 2. Analysis of expressions of Wnt signaling pathway molecules in skin tissue after Wnt5a overexpression. a-c Gene expressions of Rhoa (a), Ror2 (b) and $\mathrm{Wnt5a}$ (c) were determined by qRT-PCR. (d) Protein expressions of Wnt signaling pathway molecules were analyzed by western blot. Sample 1: telogen. Sample 2: middle anagen. Sample 3: prolonged telogen. Values are expressed as the means \pm SD $(n=3)$. *P<0.05.

Figure 3. Protein expressions of $\beta$-catenin, Frizzled and Ki67 in hair follicles after Wnt5a overexpression. C57 mice in telogen phase were depilated and injected with AdGFP (a-c) or AdWnt5a (d-f). Seven days after injection, the expressions of $\beta$-catenin (a, d), Frizzled (b, e) and $\mathrm{Ki} 67$ (c, f) were determined by immunohistochemistry. Arrowheads show the areas staining positive for expression of the indicated proteins. Scale bar $=10 \mu \mathrm{m}$.

Because the suppression of hair follicle regeneration by AdSimBC in depilated skin is similar to that observed upon AdWnt5a injection, we determined the expression patterns of $\mathrm{Wnt} / \beta$-catenin signaling molecules ( $\beta$-catenin, Dsh, Lef1), a proliferation marker (Ki67) and a hair follicle structure marker (AE15). No expression of Wnt/ $\beta$-catenin signaling molecules or proliferation marker was observed in either AdSimBC- or AdWnt5a-injected skin (Fig. 5). Neither AdSimBCnor AdWnt5a-injected skin had detectable AE15 expression (Fig. 5), which demonstrated that no new hair follicles were formed in these two groups.

\section{Wnt3a can rescue the suppression of cell proliferation by Wnt5a}

The JB6 cell line was used to perform the rescue experiment on the inhibition of hair follicle regeneration by Wnt5a. Because the adenovirus vector itself could impact the growth of JB6 cells, we utilized the Wnt5a protein and the available canonical Wnt ligand Wnt3a to perform the experiments. FACS

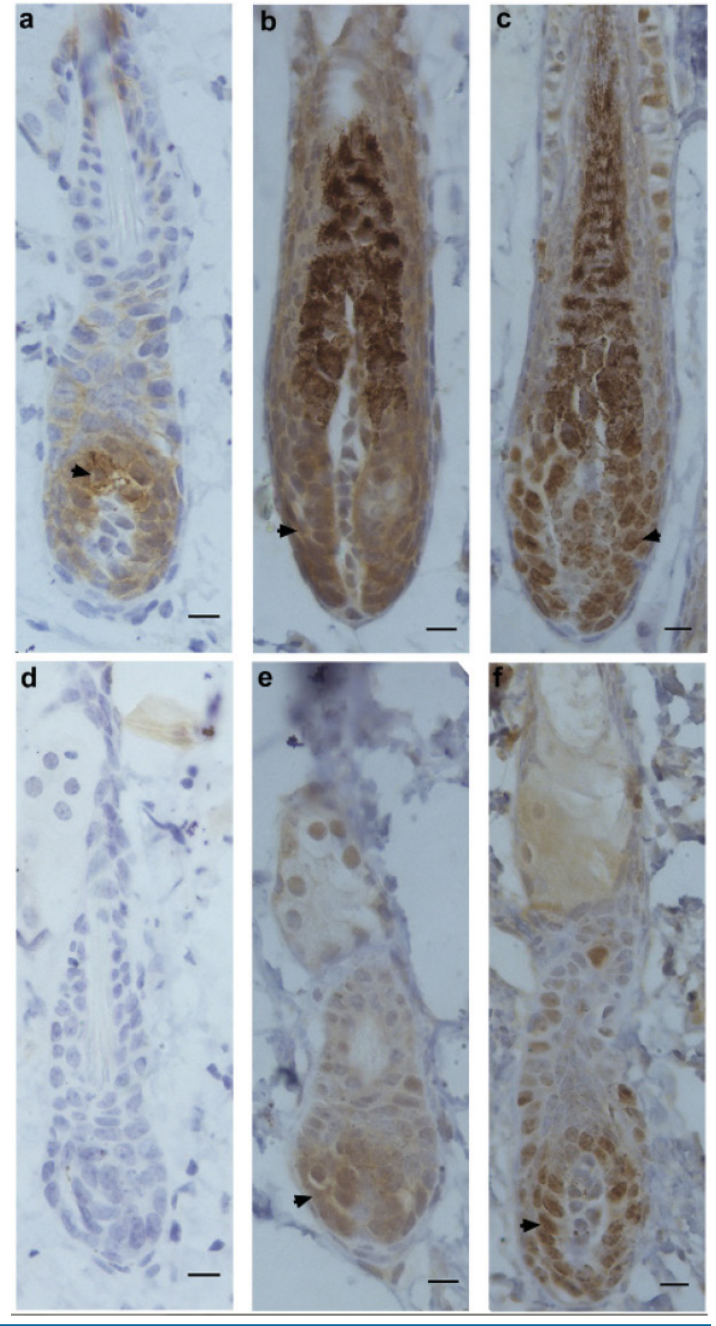


analysis revealed that JB6 cell proliferation was inhibited when Wnt5a protein was added into the culture media. When Wnt3a was added together with Wnt5a, the inhibition of cell proliferation by Wnt5a was abrogated, whereas Wnt3a added alone at the same dose had no effect on

Figure 4. The effects of SimBC on depilation-induced hair follicle regeneration. Telogen $\mathrm{C} 57$ mice were depilated and intradermally injected with AdSimBC. (a) Gross observation after AdSimBC injection. Asterisks denote the injection area. (b) HE staining of the injection area. (c) Hair type categorization of the hairs grown from the injection area. (d) Hair shafts were examined by light microscopy. (e) Hair shafts were examined by SEM. Scale bar $=50 \mu \mathrm{m}$. dP, days after AdSimBC injection.

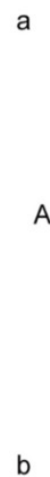

a

$b$

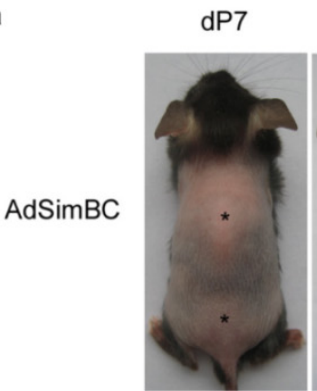

AdSimBC

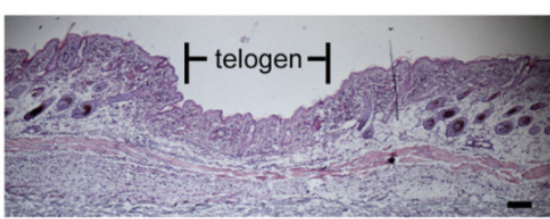

JB6 cell proliferation (Figs 6a-6e). An MTT assay obtained the same result. These data suggest that Wnt3a, which is a canonical Wnt signaling ligand, can rescue the suppression of cell proliferation by Wnt5a.

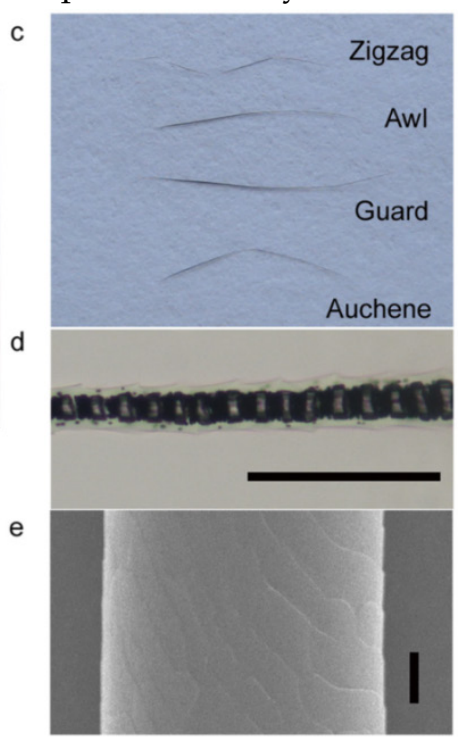

ard
Control

a

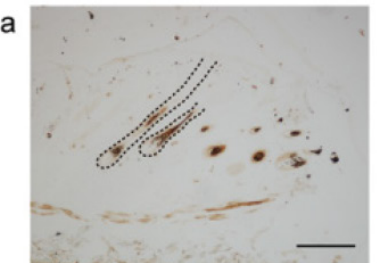

b

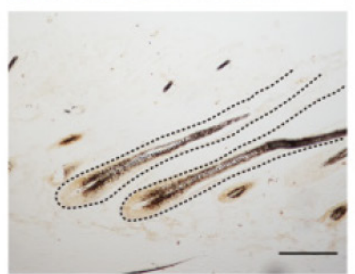

c

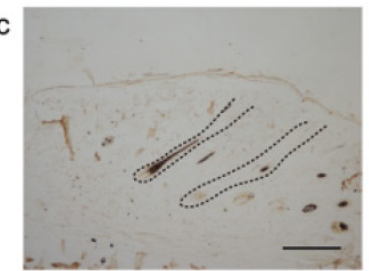

d

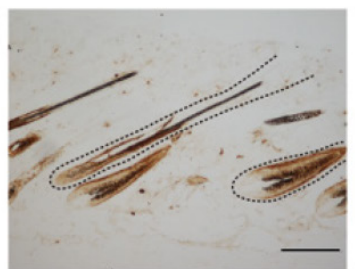

e

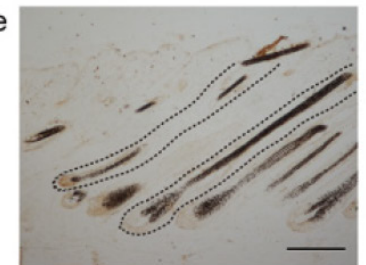

AdWnt5a
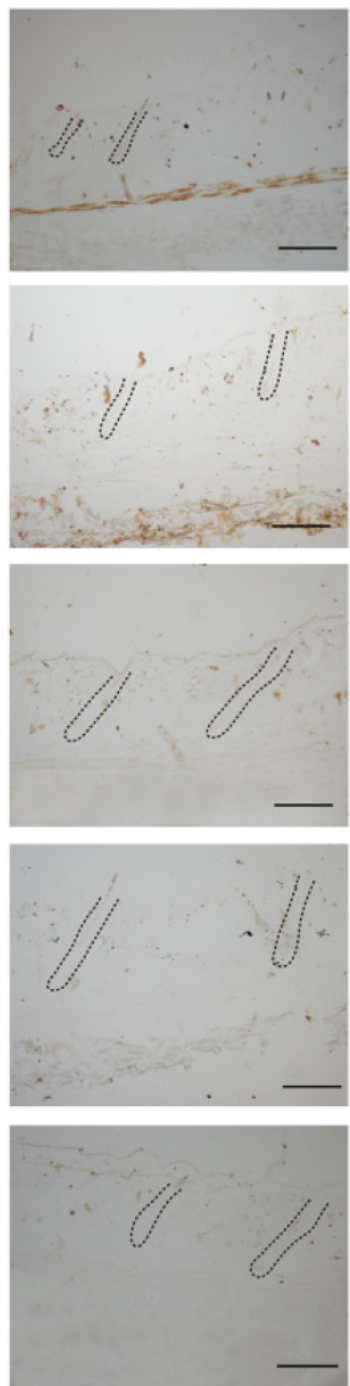

AdSimBC

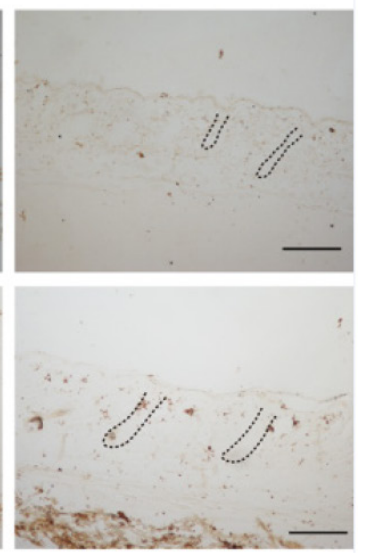

Figure 5. Comparison of the expression patterns of select markers between AdSimBC-injected skin and AdWnt5a-injected skin. Telogen C57 mice were depilated and injected intradermally with AdGFP (control), AdSimBC or AdWnt5a. Ten days later, the expressions of Dsh (a), $\beta$-catenin (b), Lefl (c), AE15 (d), and Ki67 (e) were determined by immunohistochemistry. Brown color shows the areas staining positive for expression of the indicated protein. Scale bar $=100 \mu \mathrm{m}$. 
a

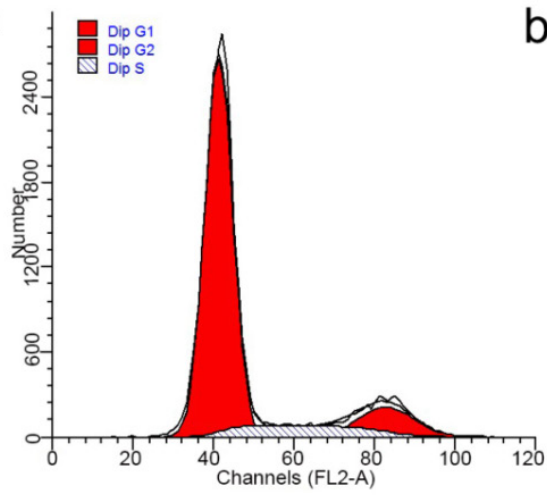

C

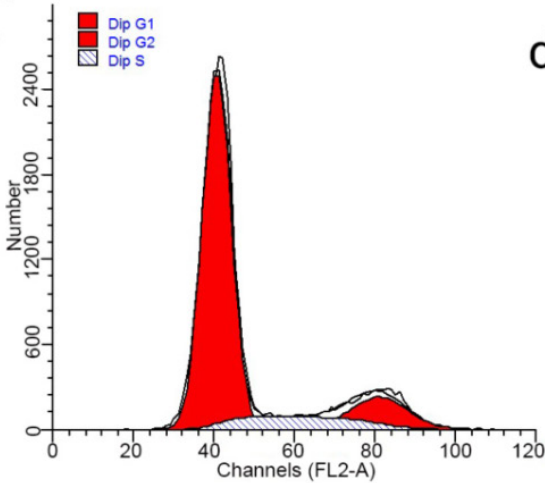

e

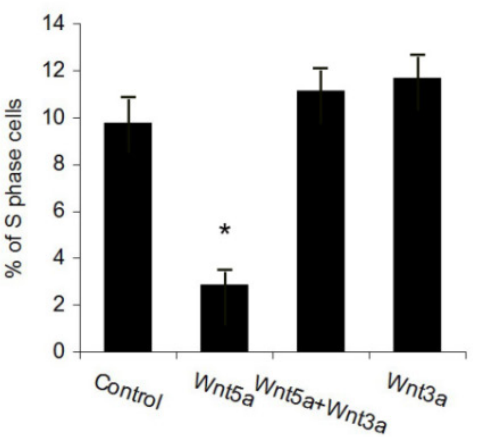

b
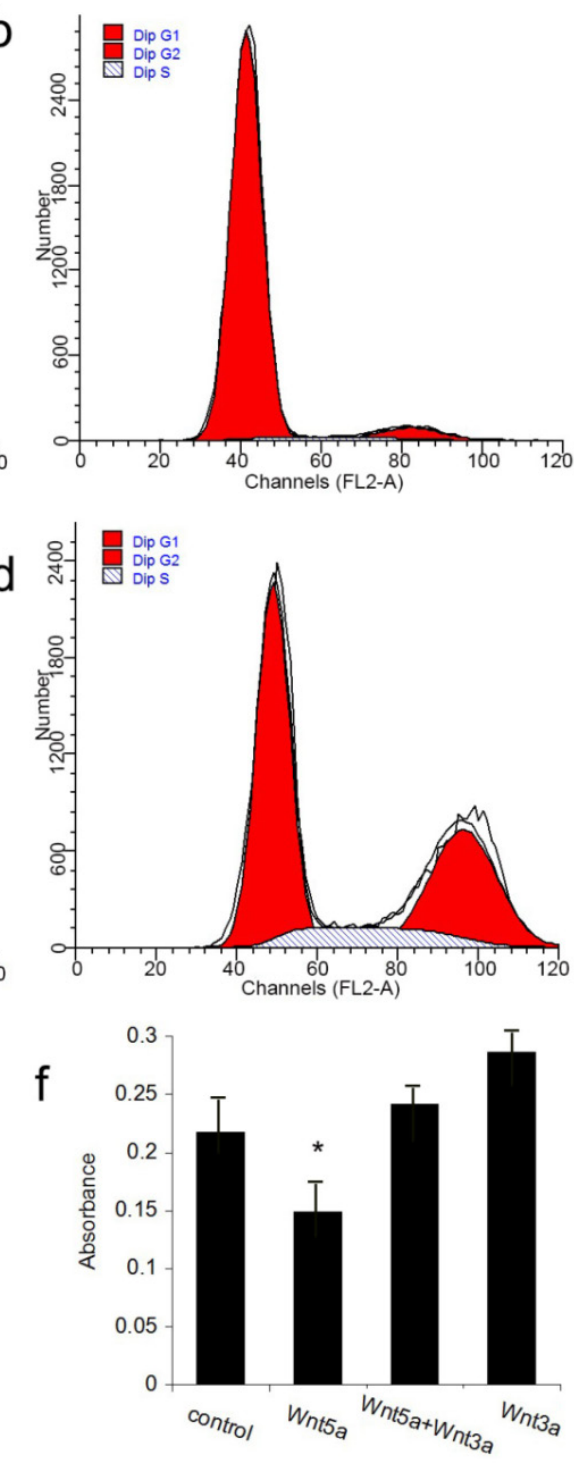

Figure 6. Effects of Wnt5a and Wnt3a on the cell proliferation of JB6 cells. Cultured JB6 cells were treated with the indicated Wnt proteins, and the proliferation statuses were determined by FACS (a-e) and MTT (f) assays. (a) Control group. (b) Wnt5a group. (c) Wnt5a+Wnt3a group. (d) Wnt3a group. (e) Quantification of percentage of $S$ phase cells in (a) to (d). (f) Quantification of MTT results. Values are expressed as the means \pm SD $(n=3)$. *P<0.05.

\section{Discussion}

Hair follicles undergo periodic regeneration, which provides a tractable model for regenerative medicine research [15]. Previous research determined that Wnt10b, Wnt7a, and Wnt7b are activators of hair follicle regeneration $[9,10]$, whereas $\mathrm{Wnt5a}$ is an inhibitor of hair follicle regeneration [13]. Our previously published data and data in this study support the model that the canonical Wnt signaling pathway and the non-canonical Wnt signaling pathways play different and even opposing roles in hair follicle regeneration. To further support this model, other Wnt ligands should be tested for their effects on hair follicle regeneration in future. Although we previously reported that the knockdown of $\beta$-catenin abrogated hair follicle regeneration, we did not determine the mechanism by which this occurred or directly compare the effects of $\beta$-catenin knockdown with the effects of overexpression of Wnt5a. In this report, by systematically comparing $\beta$-catenin knockdown to Wnt5a overexpression, we found that the overexpression of Wnt5a in skin produced a similar phenotype and gene expression patterns as did the knockdown of $\beta$-catenin in skin. Therefore, we conclude that Wnt proteins could play opposing roles in regulating hair follicle regeneration.

Wnt5a may signal through a non-canonical Wnt signaling pathway, possibly through Wnt/PCP signaling or Wnt-cGMP/Ca ${ }^{2+}$ signaling [6]. It is reported that Wnt5a regulates proliferation and apoptosis of some cells, including keratinocytes, dental follicle cells [16, 17]. Wnt5a could down-regulate $\beta$-catenin and Snail in human retinal 
pigment epithelial cells [18]. To reveal the mechanism by which Wnt5a suppresses hair follicle regeneration, we determined the expression patterns of several Wnt signaling related molecules. Rhoa is a small GTPase protein of the Rho family that is primarily associated with cytoskeleton regulation. Ror2 is a tyrosine-protein kinase transmembrane receptor. Frizzled2 is a Wnt protein receptor. All of these proteins have been reported to be involved with non-canonical Wnt signaling pathways. Our expression data imply that Wnt5a may suppress the expression of these molecules. Moreover, our published data [13] showed that the expression level of Rac2 increased markedly after Wnt5a treatment. Rac, a member of the small GTPase superfamily, is involved in the PCP signaling pathway that is activated by Wnt5a. Knockout of $\beta$-catenin or Lef1 has been reported to suppress hair follicle regeneration $[19,20]$. If Wnt5a does suppress the activation of the Wnt/ $\beta$-catenin pathway, overexpression of Wnt5a should have the same or similar phenotype and expression pattern as knockdown of $\beta$-catenin. Our data prove that this is the case in hair follicle regeneration.

During morphogenesis, hair follicles originate from the basal plate, which also generates the epidermis of the skin. The JB6 cell line was derived from primary cultures of neonatal BALB/c epidermal cells. Due to the lack of a hair follicle cell line, we performed the rescue experiment in the JB6 cell line. Both FACS and MTT assays revealed that Wnt3a could rescue the suppressive effect of Wnt5a on cell proliferation. However, the same dose of Wnt3a when administered alone did not promote the proliferation of JB6 cells. Thus, the canonical Wnt signaling pathway may have other effects on JB6 cells. A recent study reported that the prolonged overexpression of Wnt10b, another canonical Wnt ligand, induced the transformation of JB6 cells [21]. Wnt3a has also been reported to inhibit the proliferation of melanocyte cells [22]; thus, Wnt3a may also induce the transformation of JB6 cells. This possibility should be tested in future studies.

In summary, we found that Wnt5a suppressed the expression of key canonical Wnt signaling molecules and that the overexpression of Wnt5a produced a similar phenotype and expression patterns as knockdown of $\beta$-catenin. We also found that the suppressive effect of Wnt5a on cell proliferation could be rescued by Wnt3a. We conclude that Wnt5a suppresses the activation of canonical $\beta$-catenin signaling during hair follicle regeneration. Our results provide new clues not only for the research of hair follicle regeneration but also for the research of the relationship between the canonical
Wnt signaling pathway and non-canonical Wnt signaling pathways.

\section{Abbreviations}

PCP: planar cell polarity; AdWnt5a: mouse Wnt5a cDNA encoded in adenovirus vector; AdSimBC: siRNA of $\beta$-catenin encoded in adenovirus vector; AdGFP: green fluorescence protein encoded in adenovirus vector.

\section{Acknowledgements}

We thank Dr. Tong-Chuan $\mathrm{He}$ at Chicago University for adenovirus production and technical assistance. We thank Prof. Cheng-Ming Chuong at the University of Southern California for advice regarding this project. This study was supported by the National Natural Science Foundation of China (No. 31100993) and the Municipal Natural Science Foundation of Chongqing (No. cstc2015jcyjA1219).

\section{Competing Interests}

The authors declare no competing interests.

\section{References}

1. Paus R, Muller-Rover S, Van Der Veen C, et al. A comprehensive guide for the recognition and classification of distinct stages of hair follicle morphogenesis. J Invest Dermatol. 1999; 113(4): 523-32.

2. Yoshida R, Tanaka K, Amagai M, et al. Involvement of the bulge region with decreased expression of hair follicle stem cell markers in senile female cases of alopecia areata. J Eur Acad Dermatol Venereol. 2011; 25(11): 1346-50.

3. Yue $\mathrm{Z}$, Jiang $\mathrm{TX}, \mathrm{Wu} \mathrm{P}$, et al. Sprouty/FGF signaling regulates the proximal-distal feather morphology and the size of dermal papillae. Dev Biol. 2012; 372(1): 45-54.

4. Plikus MV, Mayer JA, de la Cruz D, et al. Cyclic dermal BMP signalling regulates stem cell activation during hair regeneration. Nature. 2008; 451(7176): 340-4.

5. Lei MX, Chuong CM, and Widelitz RB. Tuning Wnt signals for more or fewer hairs. J Invest Dermatol. 2013; 133(1): 7-9.

6. Semenov MV, Habas R, Macdonald BT, et al. SnapShot: Noncanonical Wnt Signaling Pathways. Cell. 2007; 131(7): 1378

7. Guo H, Xing $Y$, Liu $Y$, et al. Wnt/ $\beta$-catenin signaling pathway activates melanocyte stem cells in vitro and in vivo. J Dermatol Sci. 2016; 83(1): 45-51.

8. Andl T, Reddy ST, Gaddapara T, et al. WNT signals are required for the initiation of hair follicle development. Dev Cell. 2002; 2(5): 643-53.

9. Kandyba E and Kobielak K. Wnt7b is an important intrinsic regulator of hair follicle stem cell homeostasis and hair follicle cycling. Stem Cells. 2014; 32(4): 886-901.

10. Kandyba E, Leung Y, Chen YB, et al. Competitive balance of intrabulge $\mathrm{BMP} /$ Wnt signaling reveals a robust gene network ruling stem cell homeostasis and cyclic activation. Proc Natl Acad Sci U S A. 2013; 110(4): 1351-6.

11. Li YH, Zhang K, Ye JX, et al. Wnt10b promotes growth of hair follicles via a canonical Wnt signalling pathway. Clinical and Experimental Dermatology. 2011; 36(5): 534-540.

12. Li YH, Zhang K, Yang K, et al. Adenovirus-mediated Wnt10b overexpression induces hair follicle regeneration. J Invest Dermatol. 2013; 133(1): 42-8.

13. Xing YZ, Wang RM, Yang $\mathrm{K}$, et al. Adenovirus-mediated Wnt5a expression inhibits the telogen-to-anagen transition of hair follicles in mice. Int J Med Sci. 2013; 10(7): 908-14.

14. Luo J, Deng ZL, Luo $X$, et al. A protocol for rapid generation of recombinant adenoviruses using the AdEasy system. Nat Protoc. 2007; 2(5): 1236-47.

15. Muller-Rover S, Handjiski B, van der Veen C, et al. A comprehensive guide for the accurate classification of murine hair follicles in distinct hair cycle stages. J Invest Dermatol. 2001; 117(1): 3-15.

16. Zhang Y, Tu C, Zhang D, et al. Wnt/beta-Catenin and Wnt5a/Ca Pathways Regulate Proliferation and Apoptosis of Keratinocytes in Psoriasis Lesions. Cell Physiol Biochem. 2015; 36(5): 1890-902.

17. Sakisaka $Y$, Tsuchiya M, Nakamura T, et al. Wnt5a attenuates Wnt3a-induced alkaline phosphatase expression in dental follicle cells. Exp Cell Res. 2015; 336(1): 85-93. 
18. Kim JH, Park S, Chung $\mathrm{H}$, et al. Wnt5a attenuates the pathogenic effects of the Wnt/beta-catenin pathway in human retinal pigment epithelial cells via down-regulating beta-catenin and Snail. BMB Rep. 2015; 48(9): 525-30.

19. Huelsken J, Vogel R, Erdmann B, et al. beta-Catenin controls hair follicle morphogenesis and stem cell differentiation in the skin. Cell. 2001; 105(4): 533-45.

20. van Genderen C, Okamura RM, Farinas I, et al. Development of several organs that require inductive epithelial-mesenchymal interactions is impaired in LEF-1-deficient mice. Genes Dev. 1994; 8(22): 2691-703.

21. Lei M, Lai X, Bai X, et al. Prolonged overexpression of Wnt10b induces epidermal keratinocyte transformation through activating EGF pathway. Histochem Cell Biol. 2015; 144(3): 209-21.

22. Guo H, Yang K, Deng F, et al. Wnt3a inhibits proliferation but promotes melanogenesis of melan-a cells. Int J Mol Med. 2012; 30(3): 636-42. 